\title{
Characterization of Some Periodic Tiles by Contour Words
}

\author{
Guy Cousineau
}

Additional information is available at the end of the chapter

http://dx.doi.org/10.5772/58631

\section{Introduction}

Beauquier and Nivat [1] showed that aperiodicity cannot appear in a tiling by a polyomino using translations only : any such tiling is at least half-periodic. This is not the case when we associate a tile with one of its reflected (or rotated) images, which is a natural thing to do since, in real world, the same molecule can appear in various rotational positions or have an isomer.

Figure 1 shows a half-periodic tiling which is quite different from those with only one tile and an aperiodic one in which the reflected image appears only once.
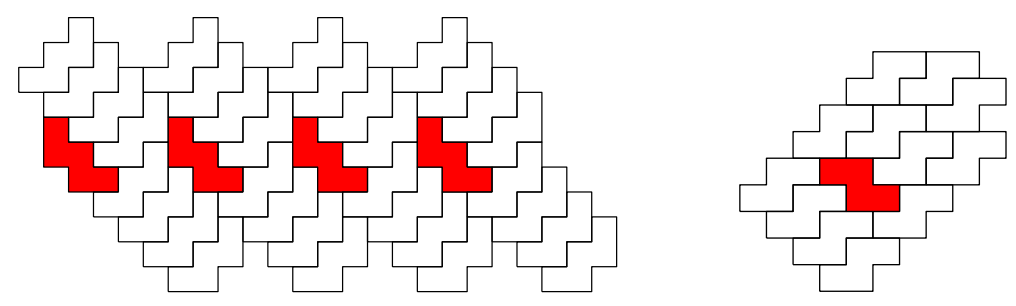

Figure 1. a half-periodic tiling and an aperiodic one

Moreover, the local structure of such tilings offers more possibilities : a tile can be surrounded by an arbitrary numbers of other tiles as can be easily inferred from the example in figure 2 .

Therefore, a general study of the tiles involved in such tilings appears to be difficult. Moreover, it is worth noting that there is a lack of notations and methods to study the decompositions of tiles and their possible surroundings.

In this paper, we provide simple word techniques to characterize some polygons that tile the plane together with one of their reflective image. We have mainly concentrated on tilings with symmetry pg [5]. Within periodic tilings involving glide reflections, this is the most interesting group to consider since it is a subgroup of all symmetry groups involving glide 


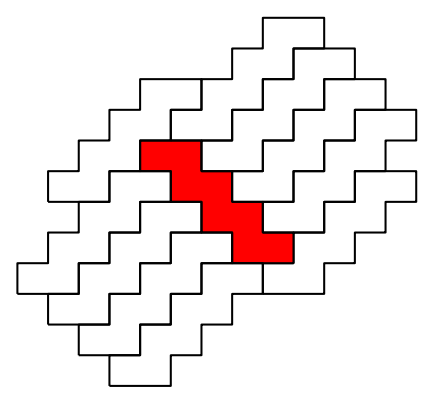

Figure 2. an unusual surrounding

reflections. However, we hope that these techniques will also be useful in the future for investigating more general problems in the spirit of [1].

\section{Polygonal tiles}

Studies similar to ours are usually placed within the framework of polyominoes. The symmetry group pg can indeed be studied in this framework since it involves only one direction of reflexion which can be made for instance horizontal by rotating the axes. However, this is unnecessarily restrictive and we shall use polygons instead. The restriction to polyominoes simplifies the description of tiles by contour words which can be expressed with a simple four letters alphabet (up,down,left,right) whereas the contour word associated with a polygon requires an infinite alphabet (the complex numbers). However, the use of polygons does not make the proofs more complex and allows for more general results.

To be precise, we shall use a notion of "polygonal tile" which in a slight extension of the notion of polygon. A polygonal tile is a sequence of points (vertices) that forms a simple closed line. The edges of a polygonal tile are the segments that join two consecutive vertices. Here, two consecutive edges can have the same direction and this distinguishes polygonal lines from what are usually called polygons. A polygon corresponds to an uncountable infinity of different polygonal lines.

When we consider possible tilings with a given polygonal line, we are only interested in tilings in which vertices of the tilings, i.e. points shared by three tiles or more, correspond to vertices of these tiles and when two tiles are adjacent, the vertices of one tile fit to vertices of the other tile. This restriction is analogous to the restriction to transformations that preserve the "grid" (points with integer coordinates) when one deals with polyominoes. It will enable us to obtain factorizations of the contour words.

\subsection{Definitions and notations}

We shall represent polygonal tiles by words on the alphabet $\mathbf{C}$ of non null complex numbers. Given a word $u$ in $\mathbf{C}^{*}$, we denote $|u|$ its length, $\Sigma(u)$ the complex sum of its letters, $R(u)$ and $I(u)$ the real and imaginary parts of $\Sigma(u)$. 
The contour word of a polygonal tile is completely defined when a starting point is chosen among its vertices. Otherwise, it is only defined modulo a circular shift of its letters The symbol $\equiv$ will denote equality modulo circular shift and the symbol $=$ will denote identity of words.

Given a word $u$, we shall denote $(-u)$ the word $u$ in which each letter is replaced by its opposite, $\bar{u}$ the word $u$ in which each letter is replaced by its conjugate, and $\widetilde{u}$ the word $u$ where the order of letters has been reversed.

These three operations commute and are involutive. Therefore, the images of a word $u$ to be considered are exactly

$$
\begin{array}{lllllllll}
u & -u & \bar{u} & \widetilde{u} & -\bar{u} & -\widetilde{u} & \widetilde{\bar{u}} & -\widetilde{\bar{u}}
\end{array}
$$

\subsection{Polygonal tiles, isometries and orientation}

The effect of isometries on contour words are the following:

- The translations leave the contour words invariants.

- The rotations by angles $\theta$ transform a contour word $u$ into the word obtained by multiplying each letter of $u$ by the complex number with modulus 1 and argument $\theta$

In particular, half-turns change $u$ into $(-u)$.

- The reflections according to a horizontal axis change $u$ into $\bar{u}$.

The other reflections are obtained by applying a rotation to $\bar{u}$.

In particular, the reflexions according to a vertical axis change $u$ into $-\bar{u}$.

- The glide reflections have the same effect as the associated reflections.

Let us also mention that a change of orientation change $u$ into $-\widetilde{u}$. As a consequence, a contour factor $v$ is centro-symmetric if and only if $v=\widetilde{v}$ ( $v$ is a palindrome).

\subsection{Periodic tiles}

All periodic tilings symmetry groups have as a subgroup p1, the group generated by two independent translations. The tiles that can produce tilings with symmetry $\mathbf{p} \mathbf{1}$ are called in [1] pseudo-hexagons and their contour in our notations have the form

$$
u v w(-\widetilde{u})(-\widetilde{v})(-\widetilde{w})
$$

We shall use this characterization as a basis to deduce characterizations for other symmetry groups. We shall start with symmetry group $\mathbf{p} 2$ for which we shall give a proof that the Conway criterion $[4,8]$ is a characterization. Then, we shall proceed to symmetry group $\mathbf{p g}$.

Beauquier and Nivat have shown that the form (1) does not only characterize the tiles that tile the plane with symmetry $\mathbf{p} \mathbf{1}$ but more generally the tiles that tile the plane by translations only. Similarly, it might well be the case that the Conway criterion characterizes the tiles that 
tile the plane by translations only together with their image by half-turn and that the criterion we give in section 4.2 characterizes the tiles that tile the plane by translations only together with their image by reflection. However we have not been able to obtain such a result. This remains, to our knowledge, a conjecture.

\subsection{Translation adjacency}

A polygonal tile can be made adjacent to a translated image of itself only if its contour has both a factor $x$ and a factor $(-\widetilde{x})$. These two factors are necessarily disjoint because otherwise we have $x=y z$ and $(-\widetilde{x})=z u$, which implies $(-\widetilde{z})(-\widetilde{y})=z u$ and finally $(-\widetilde{z})=z$. Such a word cannot be a factor of a contour word since this implies $\Sigma(z)=0$.

Therefore, a tile that can be made adjacent to a translated image of itself has the shape $x y(-\widetilde{x}) z$ and the translation associated with the superposition of the factors $x$ and $(-\widetilde{x})$, which we shall denote by $T_{x}$, is defined by the complex number $\Sigma(y)=-\Sigma(z)$. Such a decomposition $x y(-\widetilde{x}) z$ of a contour word will be called a T-decomposition. A T-decomposition will be said exact when $x$ is maximal for translation $T_{x}$ i.e. when the two following conditions are satisfied:

- $y$ cannot be factorized into $y=y_{1} y^{\prime}\left(-\widetilde{y_{1}}\right)$ with $\left|y_{1}\right| \neq 0$

- $z$ cannot be factorized into $z=z_{1} z^{\prime}\left(-\widetilde{z_{1}}\right)$ with $\left|z_{1}\right| \neq 0$

A T-decomposition $x y(-\widetilde{x}) z$ is therefore exact if and only if the contact of the corresponding tile with its image by translation $T_{x}$ is exactly $(-\widetilde{x})$.

\subsection{Half-turn adjacency}

A tile that can be made adjacent to its image by a half-turn must have both a factor $x$ and a factor $\widetilde{x}$. These factors could be disjoint, overlapping or identical. We shall show that the only case we have to consider is the case where $x$ is a palindromic factor equal to $\widetilde{x}$. Such a palindromic factor will be said exact if it is not the center of a bigger palindrome.

To clarify the discussion, let us denote by $h t$ the corresponding half-turn, by A the starting point of factor $x$, by B its end point, and by $\mathrm{A}^{\prime}$ and $\mathrm{B}^{\prime}$ the images of $\mathrm{A}$ and $\mathrm{B}$ by halft-turn $h t$.

The case where $x$ and $\tilde{x}$ are identical correspond to $A^{\prime}=B$ and $B^{\prime}=A$. It is not possible to have $A^{\prime}=A$ and $B^{\prime}=B$ because a half-turn has only one fixpoint.

The case where the factors $x$ and $\tilde{x}$ are disjoint correspond to a situation where the order between the four considered points are either $A, B, A^{\prime}, B^{\prime}$ or $A, B, B^{\prime}, A^{\prime}$. However the former case is impossible because, since half-turns are involutive, we have $h t\left(A^{\prime}\right)=A$ and $h t\left(B^{\prime}\right)=$ $B$. Therefore the image of $A B^{\prime}$ is $A^{\prime} B$ a factor strictly contained in $A B^{\prime}$ which is impossible since half-turns are isometries and preserve the lengthes. We are left with the latter case where the order is $A, B, B^{\prime}, A^{\prime}$. Let us denote by $w$ the factor $B B^{\prime}$. Since the image by $h t$ of $B B^{\prime}$ is $B^{\prime} B$, if $h t$ is a tiling transformation, then $w$ must be a palindrome and $A A^{\prime}=x w \tilde{x}$ is a bigger palindrome that includes both $x$ and $\tilde{x}$ and is its own image under $h t$.

Now, in the last case where factors $x$ and $\tilde{x}$ are overlapping, we can eliminate the case where $A B$ is included in $A^{\prime} B^{\prime}$ or $A^{\prime} B^{\prime}$ included in $A B$ using the length argument and we are left 
with the cases $A, A^{\prime}, B, B^{\prime}$ and $A, B^{\prime}, B, A^{\prime}$. Here again, the former can be eliminated by the length argument. In the latter, the factor $x$ is decomposed into $x=u w$ where $w$ corresponds to $B B^{\prime}$ and must therefore be a palindrome and again $x$ and $\widetilde{x}$ are part of a bigger palindromic factor $u w \widetilde{u}$ which is its own image under $h t$.

\subsection{Reflection adjacency}

We are mainly interested here in symmetry pg which has only one direction of (glide) reflexion. Also, if we study tilings by a tile and one of its images by reflection, we are also in a situation where one reflection direction is privileged. Therefore, it will be convenient to consider that this unique reflection direction is horizontal. This will enable us to express reflections or glide reflections by applying the complex conjugation operation to its contour word.

A tile that can be made adjacent to its image by reflection or glide reflection must therefore possess both a factor $x$ and a factor $\bar{x}$. These factors can be identical, overlapping or disjoint. When they are identical, $x$ contains only real numbers: it is made of a sequence of horizontal segments. This cannot occur in symmetry pg because glide reflexions have no fixpoints.

When the two factors $x$ and $\bar{x}$ overlap, let us denote by $u$ their common factor. We have $x=w u$ and $\bar{x}=u w^{\prime}$ and thus $u w^{\prime}=\overline{w u}$. If we are in symmetry pg, the transformation that transforms $x$ into $\bar{x}$ is a glide reflection $g r$ which can be decomposed into an horizontal reflection $r$ and an horizontal translation $t$. The effect of the glide reflection $g r$ on factor $x$ is completely described by the complex number $\Sigma(w)$ which has an imaginary part which correspond to the effect of the reflection $r$ and a real part which corresponds to the effect of the translation $t$. The complex number $\Sigma(w \bar{w})$, which is a real number, corresponds to applying twice the horizontal translation $t$.

Let us assume $|\bar{w}|<|u|$. This implies $u=\bar{w} u^{\prime}$ and $x=w \bar{w} u^{\prime}$. This implies that $x$ overlaps with its image obtained by applying twice the glide reflection $g r$ or equivalently twice the translation $t$. But this is impossible in symmetry pg because this translation belongs to the symmetry group (as the composition of the glide reflection with itself) and in a periodic tiling, an element of a a tile contour cannot overlap with its image by translation. The only possibility is therefore $|\bar{w}| \geq|\bar{u}|$. In that case, $x$ factorizes into $x=\bar{u} v u$ and $\bar{x}$ is equal to $u \overline{v u}$. The tile contour has then a factor $\bar{u} v u \overline{v u}$.

Finally, the two factors $x$ and $\bar{x}$ can be disjoint. The tile contour has shape $x y \bar{x} z$ where $y$ may be empty. We can note that the overlapping case is a special case of this last one, where $\bar{u}$ and $u$ play the parts of $x$ and $\bar{x}$.

\section{Tiles for symmetry $\mathrm{p} 2$}

\subsection{Presentation of symmetry group $\mathrm{p} 2$}

The symmetry group p2 can be generated by three half-turns or by one half-turn and two translations. The latter is more convenient since it leads to a presentation which allows for a characterization of normal forms by a canonical rewriting system. 
If we denote the generators by $T$ (the half-turn) and $X, Y$ (the translations), the equations are:

$$
X Y=Y X, T^{2}=I, T X T=X^{-1}, T Y T=Y^{-1}
$$

We give below the associated canonical rewriting system [6] together with an example:

$$
\begin{array}{rlrl}
\mathrm{T}^{-1} & \rightarrow \mathrm{T} & \mathrm{TY}^{-1} & \rightarrow \mathrm{YT} \\
\mathrm{TX}^{-1} & \rightarrow \mathrm{XT} & \mathrm{X}^{-1} \mathrm{Y} & \rightarrow \mathrm{YX}^{-1} \\
\mathrm{~T}^{2} & \rightarrow 1 & \mathrm{XY} & \rightarrow \mathrm{YX} \\
\mathrm{X}^{-1} \mathrm{Y}^{-1} & \rightarrow \mathrm{Y}^{-1} \mathrm{X}^{-1} & \mathrm{XY}^{-1} & \rightarrow \mathrm{Y}^{-1} \mathrm{X} \\
\mathrm{TX} & \rightarrow \mathrm{X}^{-1} \mathrm{~T} & \mathrm{TY} & \rightarrow \mathrm{Y}^{-1} \mathrm{~T}
\end{array}
$$

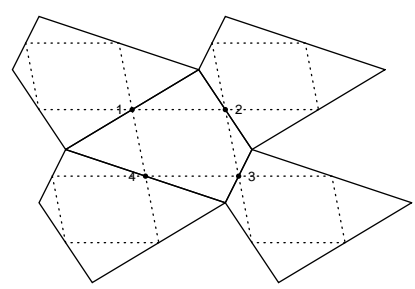

The normal forms are of two sorts: $Y^{p} X^{q}$ (translations) and $Y^{p} X^{q} T$ (half-turns)

It thus appears that a tile is adequate for a (isohedral) tiling with symmetry $\mathbf{p} 2$ if and only if this tile joined to its image by the half-turn $T$ forms an adequate tile for symmetry group $\mathbf{p} 1$. This observation explains in a simple way why the Conway criterion is a necessary condition in section 3.3.

\subsection{The Conway criterion}

A polygonal tile satisfies the Conway criterion if and only if it has the shape $u v w(-\widetilde{u}) x y$ where $v, w, x$ and $y$ are palindromes. It is easy to see that if we choose any of its palindromic factors and join it with its half-turn image according to this factor, we obtain a pseudo-hexagon.

Take for instance factor $y$. We obtain

$$
u v w(-\widetilde{u}) x(-u)(-v)(-w) \widetilde{u}(-x)
$$

or, with a left shift

$$
v w(-\widetilde{u}) x(-u)(-v)(-w) \widetilde{u}(-x) u
$$

Taking $z=(-\widetilde{u}) x(-u)$, we have $-z=\widetilde{u}(-x) u$ and the polygon contour appears as:

$$
v w z(-v)(-w)(-z)
$$

where $v, w$ et $z$ are palindromes.

This is a specific form of pseudo-hexagon which is invariant by half-turn. 


\subsection{Conway criterion characterizes $\mathrm{p} 2$}

Reciprocally, let us state the condition for a tile to produce a pseudo-hexagon when joined with its image by half-turn.

First, as mentioned in section 2.5, the tile's contour must have a palindromic factor and therefore have shape $x y$ where $y$ is a palindrome. When the tile is joined to its image by the half-turn, the resulting tile has contour $x(-x)$ and this contour must be that of a pseudo-hexagon. Therefore, there must exist words $u, v$ and $w$ such that:

$$
x(-x) \equiv u v w(-\widetilde{u})(-\widetilde{v})(-\widetilde{w})
$$

The word $x(-x)$ must thus be identical to a shift of $u v w(-\widetilde{u})(-\widetilde{v})(-\widetilde{w})$.

If $x(-x)=u v w(-\widetilde{u})(-\widetilde{v})(-\widetilde{w})$, then $x=u v w$ and $-x=(-\widetilde{u})(-\widetilde{v})(-\widetilde{w})$, which is equivalent to $x=(\widetilde{u})(\widetilde{v})(\widetilde{w})$. We thus have $u v w=(\widetilde{u})(\widetilde{v})(\widetilde{w})$ which implies $u=\widetilde{u}, v=\widetilde{v}$ and $w=\widetilde{w}$. The words $u, v$ et $w$ are palindromes and the primitive tile $x y$ is equal to $u v w y$ where $u, v, w$ and $y$ are palindromes. We are in a special case where the tile is made of four centro-symmetric factors.

If $x(-x) \equiv u v w(-\widetilde{u})(-\widetilde{v})(-\widetilde{w})$ but $x(-x) \neq u v w(-\widetilde{u})(-\widetilde{v})(-\widetilde{w})$, by shifting if necessary the names $u, v, w, \widetilde{u}, \widetilde{v}, \widetilde{w}$, we can assume without lost of generality that $u$ can be decomposed into $u=u_{1} u^{\prime} u_{2}$ in such a way that $\left|u_{1}\right|=\left|u_{2}\right|$ and either

$$
x(-x)=u^{\prime} u_{2} v w\left(-\widetilde{u_{2}}\right)\left(-\widetilde{u}^{\prime}\right)\left(-\widetilde{u_{1}}\right)(-\widetilde{v})(-\widetilde{w}) u_{1}
$$

or

$$
x(-x)=u_{2} v w\left(-\widetilde{u_{2}}\right)\left(-\widetilde{u}^{\prime}\right)\left(-\widetilde{u_{1}}\right)(-\widetilde{v})(-\widetilde{w}) u_{1} u^{\prime}
$$

The second case differs from the first by the orientation only. Therefore, it is sufficient to deal with the first case.

We have $x=u^{\prime} u_{2} v w\left(-\widetilde{u_{2}}\right)$ and $-x=\left(-\widetilde{u}^{\prime}\right)\left(-\widetilde{u_{1}}\right)(-\widetilde{v})(-\widetilde{w}) u_{1}$, which is equivalent to $x=$ $\widetilde{u^{\prime}} \widetilde{u_{1}} \widetilde{v w}\left(-u_{1}\right)$, and thus $u^{\prime} u_{2} v w\left(-\widetilde{u_{2}}\right)=\widetilde{u^{\prime}} \widetilde{u_{1}} \widetilde{v} \widetilde{w}\left(-u_{1}\right)$.

This implies $u^{\prime}=\widetilde{u}^{\prime}, u_{2}=\widetilde{u_{1}}, v=\widetilde{v}$ et $w=\widetilde{w}$.

The primitive tile's contour can thus be written $u^{\prime} u_{2} v w\left(-\widetilde{u_{2}}\right) y$ or by shifting it to the left $u_{2} v w\left(-\widetilde{u_{2}}\right) y u^{\prime}$ where $v, w, y$ and $u^{\prime}$ are palindromes which corresponds exactly to the Conway criterion.

\section{Tiles for symmetry $\mathrm{pg}$}

\subsection{Presentation of symmetry group pg}

The symmetry group pg can be generated with two glide reflexions with parallel mirrors and equal associated translations. The presentation has just one equation $G_{1}{ }^{2}=G_{2}{ }^{2}$ where $G_{1}$ and $G_{2}$ are the two glide reflections. 
It can also be generated by a single glide reflection $G$ and a translation $X$ perpendicular to the mirror of $G$. The presentation still has just one equation which is $X G X=G$. This second presentation is more interesting. We give below the associated canonical rewriting system [6] together with an example:

$$
\begin{gathered}
\mathrm{GX} \rightarrow \mathrm{X}^{-1} \mathrm{G} \\
\mathrm{GX}^{-1} \rightarrow \mathrm{XG} \\
\mathrm{G}^{-1} \mathrm{X} \rightarrow \mathrm{X}^{-1} \mathrm{G}^{-1} \\
\mathrm{G}^{-1} \mathrm{X}^{-1} \rightarrow \mathrm{XG}^{-1}
\end{gathered}
$$

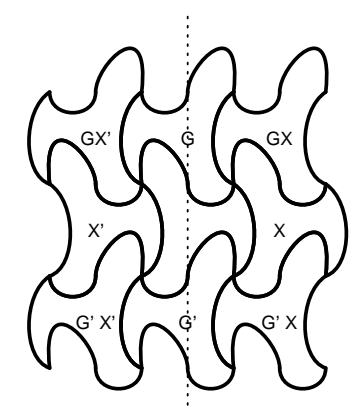

The normal forms are $X^{p} G^{2 q}$ (translations) and $X^{p} G^{2 q+1}$ (glide reflections). Taking $Y=G^{2}$ ( $Y$ is a translation parallel to the mirror of $G$ ), the normal forms can be described as $X^{p} Y^{q}$ (translations) and $X^{p} Y^{q} G$ (glide reflections).

This permits to see that a tile is adequate for symmetry pg if and only if this tile joined to its image by the glide reflexion $G$ forms an adequate tile for symmetry group $\mathbf{p} 1$.

\subsection{Criterion for pg tiles}

We are going to show that a tile is adequate for symmetry pg if and only if it has one of the shapes

$$
\begin{aligned}
& u v w \bar{v} \bar{u}(-\widetilde{w}) \\
& u \bar{u} w v \bar{v}(-\widetilde{w})
\end{aligned}
$$

In both cases, one of the factors may be empty.

Let us first show that we obtain a pseudo-hexagon when we make such a tile adjacent to its reflective image using factor $u$ or $v$. We shall give the proof for the first form: the second one is similar.

Since the factors $u$ and $v$ play similar parts, we shall use the reflection according to factor $u$. The reflected tile has contour $\bar{u} \bar{v} \bar{w} v u(-\overline{\bar{w}})$ in the reverse orientation or if we give the right orientation

$$
(-\widetilde{\bar{u}}) \bar{w}(-\widetilde{u})(-\widetilde{v})(-\widetilde{\bar{w}})(-\widetilde{\bar{v}})
$$


Gluing together the primitive tile and its reflected image using factors $\bar{u}$ and $(-\widetilde{\bar{u}})$, we obtain a polygon with contour

$$
u v w \bar{v} \bar{w}(-\widetilde{u})(-\widetilde{v})(-\widetilde{\bar{w}})(-\widetilde{\bar{v}})(-\widetilde{w})
$$

Taking $x=w \bar{v} \bar{w}$ which implies $-\widetilde{x}=(-\widetilde{\bar{w}})(-\widetilde{\bar{v}})(-\widetilde{w})$, this contour can be written

$$
u v x(-\widetilde{u})(-\widetilde{v})(-\widetilde{x})
$$

which is a pseudo-hexagon. The translation $X$ corresponds to the complex number $\Sigma(u v)$ and the translation $Y$ to the complex number $\Sigma(v x)=\Sigma(v w \bar{v} \bar{w})$

\subsection{The criterion characterizes pg tiles}

Let us assume that some tile with contour $m_{1}$ glued to its reflected image produce a pseudo-hexagon. The conditions for a tile to fit its reflected image have been stated in section 2.6.

In all cases suitable for $\mathbf{p g}$, we have

$$
m_{1}=x y \bar{x} z
$$

and the obtained polygon has contour

$$
m_{2}=x y(-\widetilde{\bar{z}})(-\widetilde{x})(-\widetilde{\bar{y}}) z
$$

We can also use one more information that comes from the structure of group pg. When we compose any glide reflection belonging to the symmetry group pg with itself, we obtain a translation that belongs to the symmetry group. Therefore, if in the contour $m_{1}$, the factor $\bar{x}$ is the image of the factor $x$ in some glide reflection, in the contour $m_{2}$, the factor $(-\widetilde{x})$ is image of the factor $x$ in the corresponding translation. Note that it is written $(-\widetilde{x})$ and not $x$ because it is read in the reverse order in the contour of $m_{2}$. However, even if $x$ is an exact factor for the glide reflection, it is not necessarily the case that it is an exact factor for the corresponding translation: we can only assume that $x$ is part of the exact factor for the translation. This distinction will lead to the two possible forms for pg tiles.

If we assume that $x$ is an exact factor for the translation then $x$ appears in the pseudo-hexagon decomposition of $m_{2}$. Therefore, $m_{2}$ can be written as

$$
m_{2}=x u v(-\widetilde{x})(-\widetilde{u})(-\widetilde{v})
$$


and therefore, we must have

$$
u v=y(-\widetilde{\bar{z}})
$$

and

$$
(-\widetilde{u})(-\widetilde{v})=(-\widetilde{\bar{y}}) z
$$

and in the special case where $z$ is empty (the case where $y$ is empty is similar), we get

$$
u v=y
$$

and

$$
(-\widetilde{u})(-\widetilde{v})=(-\widetilde{\bar{y}})
$$

We shall first deal with this special case. The equation (10) can be rephrased into

$$
y=\bar{v} \bar{u}
$$

and we have

$$
u v=\bar{v} \bar{u}
$$

This equation has two types of solutions (see lemma 5.1):

- The general solution:

$u=(w \bar{w})^{p} w$ et $v=\bar{w}(w \bar{w})^{q}$

At this point, we have to use the fact that one of the translations which associate $(-\widetilde{u})$ to $u$ or $(-\widetilde{v})$ to $v$ is a vertical translation. This fact implies $R(u)=R(v)=R(x)$ and therefore $p=q$ and $u=\bar{v}$.

The tile has shape $m_{1}=x \bar{x}(-\widetilde{u})(-\widetilde{\bar{u}})$ or, stating $y=(-\widetilde{u}), m_{1}=x \bar{x} y \bar{y}$ (see figure 3 ).

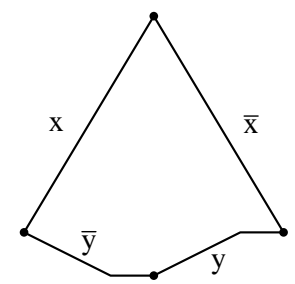

Figure 3. Special case of the first form 
- The solution on reals:

$u=a^{p}$ et $v=a^{q}$.

For the same reason, we must have $p=q$. This is only a subcase of the previous one.

Now we go back to equations (7) and (8). Here again, we shall use the fact that one of the translations corresponding to $u$ and $v$ is a vertical one. Let us assume that the vertical translation is associated with $u$. Let us also assume that $R(x) \geq 0$ which implies $R(\bar{x})=$ $R(x) \geq 0$. This is just to ensure that all the vertical projections as they are defined below do exist. The case $R(x) \leq 0$ is similar with $x$ and $z$ having inverted roles.

Let us denote by $1,2,3,4,5,6$ the vertices that bound the factors $x, y,(-\widetilde{\bar{z}}),(-\widetilde{x}),(-\widetilde{\bar{y}})$ and $z$. Let us also denote $2^{\prime}, 3^{\prime}$ the images of 2,3 in the vertical translation and $5^{\prime}, 6^{\prime}$ the images of 5 et 6 in its inverse (see figure 4 ).

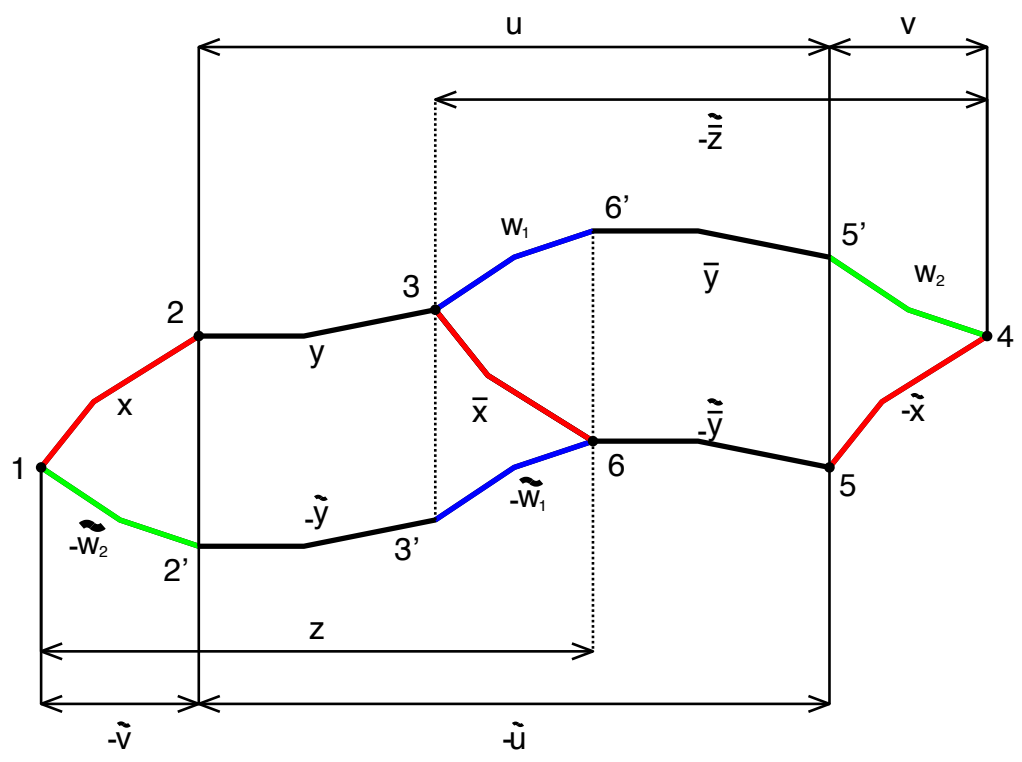

Figure 4. General case 1

The factor $u$ has extremities 2 and $5^{\prime}$ and the factor $v$ has extremities $5^{\prime}$ et 4 . Let us denote by $w_{1}$ the factor with extremities 3 and $6^{\prime}$ and $w_{2}$ the factor with extremities $5^{\prime}$ and 4 . The factor with extremities $3^{\prime}$ and $2^{\prime}$ is equal to $(-\widetilde{y})$ and therefore we have

$$
(-\widetilde{\bar{z}})=w_{1} \bar{y} w_{2}
$$

We also have

$$
z=\left(-\widetilde{w_{1}}\right)(-\widetilde{y})\left(-\widetilde{w_{2}}\right)
$$


since the factors $\left[3,6^{\prime}\right],\left[6^{\prime}, 5\right]^{\prime}$ et $\left[5^{\prime}, 4\right]$ are images by translation of factors $\left[3^{\prime}, 6\right],[6,5]$ et $\left[1,2^{\prime}\right]$.

This last equality can also be written

$$
(-\widetilde{\bar{z}})=\overline{w_{2}} \bar{y} \overline{w_{1}}
$$

and we have

$$
w_{1} \bar{y} w_{2}=\overline{w_{2}} \bar{y} \overline{w_{1}}
$$

which is an instance of equation

$$
x y z=\bar{z} y \bar{x}
$$

Fortunately, we shall not have to solve this equation in full generality because we can use the fact that $R\left(w_{1}\right)=R\left(w_{2}\right)$ i.e. in the above equation $R(x)=R(z)$. We shall show that in this case $x=\bar{z}$. If this is not the case then either $x=\bar{z} u$ for some $u$ or $z=\bar{x} v$ for some $v$. These two cases being symmetrical, we shall deal with the first one only. Since $x$ and $z$ have equal real projections, $R(u)=0$ ( $u$ is a pure imaginary number). On the other hand, we have $\bar{z} u y z=\bar{z} y z \bar{u}$ which implies $I(\bar{z} u y z)=I(\bar{z} y z \bar{u})$ which is equivalent to $I(u)=I(\bar{u})$. But this is possible only if $I(u)=0$ which, since we already know that $R(u)=0$, implies $\Sigma(u)=0$ i.e. $u$ would be a closed (looping) factor which cannot exist in a contour word except for the entire contour word. So, we do have $x=\bar{z}$.

So, this shows that in our tile, $w_{1}=\overline{w_{2}}$ and the contour word can finally be written, setting $w=\left(-\widetilde{w_{2}}\right)$,

$$
w x y \bar{x} \bar{w}(-\widetilde{y})
$$

which is of the required shape (form (2).

Notice that if we do not use the fact that one of the translations is vertical, then the real projections of $w_{1}$ and $w_{2}$ need not be equal. In that case, we have to consider also solutions such as:

- $w_{1}=(w \bar{w})^{p} w,(-\widetilde{y})=(w \bar{w})^{n}, w_{2}=\bar{w}(w \bar{w})^{q}$

- $w_{1}=(w \bar{w})^{p},(-\widetilde{y})=(w \bar{w})^{n} w, w_{2}=(\bar{w} w)^{q}$ 

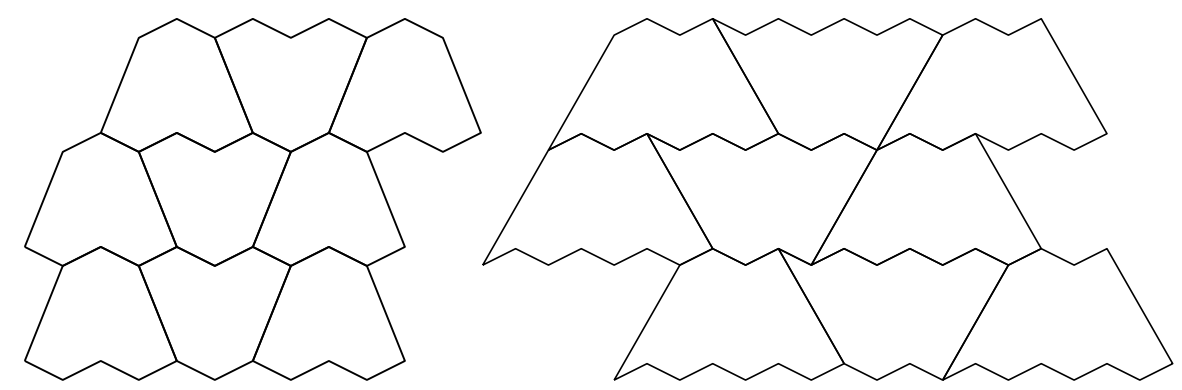

Figure 5. Two tilings with non-pg symmetry

These solutions with $p \neq q$ correspond to tiles that produce tilings with other symmetries than pg or no symmetry at all as those on figure 5 which are only half-periodic or on figure 1 where aperiodicity appears.

Now, we go back to the case where the factor $x$ of $m_{2}$ in equation 5 is not an exact factor of the horizontal translation. This means that $x$ is included inside some translation factor $a x b$ where $a b$ is non-empty. We assume that the glide reflection maps $x$ to $\bar{x}$ and $x$ is an exact factor for this glide reflection. If we apply again the glide reflection to $\bar{x}$, we obtain the image of $x$ in the horizontal translation which has to belong to the part of $m_{2}$ which is not in $m_{1}$ because, otherwise, $m_{1}$ would overlap with its image in the horizontal translation.

Let us show that if $a$ and $b$ are both non-empty, they cannot both belong to the contour of $m_{1}$. If $a$ is non-empty, its image in the glide reflection does not belong to the contour of $m_{1}$ because otherwise, $x$ would not be an exact factor. But then, for the same reason, the image of $a$ under the horizontal translation cannot belong to the contour of $m_{2}$ outside $m_{1}$, so it has to belong to $m_{1}$. The situation is the same for $b$. Consequently, if we consider the images of $x, a, b$ are such that the fist one should be outside $m_{1}$ and the two others inside $m_{1}$. But then we would have to assume that the image of $x$ in the horizontal translation is all the part of $m_{2}$ that is outside $m_{1}$ and $m_{1}$ would have to be equal to $x \bar{x}$ and $a$ and $b$ would both be empty.

So, one of the factors $a$ or $b$, if they are non-empty have to be outside the contour of $m_{1}$ and therefore belong to the part of $m_{2}$ that is outside $m_{1}$. Let us say, it is $b$. This implies that, in the contour of $m_{1}$, the two factors $x$ and $\bar{x}$ are adjacent and $b$ has to be the image of $a$ in the glide reflection as shown on figure 6 .

$$
m_{1}=a x \bar{x}(-\widetilde{a}) z
$$

and

$$
m_{2}=a x(-\widetilde{\bar{a}})(-\widetilde{\bar{z}}) \bar{a}(-\widetilde{x})(-\widetilde{a}) z
$$

We know that the factor $a x(-\widetilde{\bar{a}})$ is exact for the horizontal translation. So, we can make explicit the fact that $m_{2}$ is a pseudo-hexagon by factorizing $(-\widetilde{\bar{z}})$ into $(-\widetilde{\bar{z}})=u v$ with $z=$ 


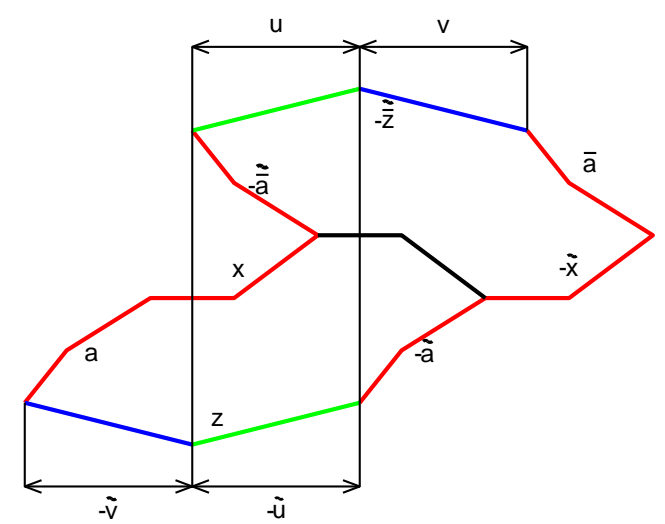

Figure 6. General case 2

$(-\widetilde{u})(-\widetilde{v})$. The two definitions of $z$ lead us to the equation

$$
u v=\bar{v} \bar{u}
$$

and we use the fact that one of the translations associated to factors $u$ and $v$ is vertical to deduce that $R(u)=R(v)$. Therefore $u=\bar{v}$ and we can conclude that

$$
m_{1}=\operatorname{ax} \bar{x}(-\widetilde{a})(-\widetilde{u})(-\widetilde{\bar{u}})
$$

which corresponds to the form 3.

This completes our proof. It is clear from the examples of figure 5 that the main result presented here is only a small step toward the characterization of polygons that tile the plane together with one of their reflected images because our proofs are highly dependent on the specificities of symmetry pg.. A plausible conjecture is that a polygon has this property if and only if, suitably oriented, its contour has a factorization of form 2 or 3 . But the contour word techniques that we have used here will certainly require further development in order to tackle with such a conjecture.

\section{Technical lemmas}

\section{Solutions of the equation $x \equiv \bar{x}$}

This section studies the solutions of the equation $x \equiv \bar{x}$. This equation where the symbol $\equiv$ denotes equality modulo circular shift corresponds to two equations using equality on words.

1. $x=\bar{x}$

In that case, each letter of $x$ must be its own conjugate. The word $x$ is made uniquely of reals. 
2. $x=u v$ for two non empty words $u$ et $v$ and $u v=\bar{v} \bar{u}$.

This case is treated by lemma 5.1.

To study such equations, the alphabet of complex numbers divides naturally between reals that are their own conjugate and non reals which have a conjugate distinct from themselves. The lemmas presented below are very similar to those presented in [7] for ordinary equations on words but are slightly more complex due to the partition of the alphabet. The equations will have two kinds of solutions: solutions on $R^{*}$ which are the solutions of the considered equations where we drop out the conjugation operations and the general solutions.

Lemme 5.1. The solutions of equation $u v=\bar{v} \bar{u}$ are

$$
u=w^{m}, v=w^{n}
$$

where $w \in R^{*}$ and

$$
u=(w \bar{w})^{m} w, v=\bar{w}(w \bar{w})^{n}
$$

where $w \in C^{*}$.

To prove this lemma, we shall have to prove also the following one:

Lemme 5.2. The solutions of equation $u v=\bar{v} u$ are

$$
u=w^{m}, v=w^{n}
$$

where $w \in R^{*}$ and

$$
u=(w \bar{w})^{m} w, v=(\bar{w} w)^{n}
$$

The proofs of these two lemmas can be done simultaneously by induction on $|u v|$. We shall assume $|u v| \geq 2$. The base case will correspond to $|u v|=2$ and $|u v|=3$.

- If $|u v|=2, u$ and $v$ are letters and $u=\bar{v}$ in the three cases. This leads to solutions $u=\bar{u}=v=\bar{v}$ on reals and $u=\bar{v}$ on non real complex numbers for lemma 5.1. For lemma 5.2, there is no solution on non real complex numbers.

- If $|u v|=3$, we have either $u=a b$ et $v=c$ or $u=a, v=b c$ where $a, b, c$ are letters. For lemma 5.1 , the two hypotheses lead to $a=\bar{a}=b=\bar{b}=c=\bar{c}$ and we have only the solutions $u=a, v=a a$ et $u=a a, v=a$ where $a$ is a real letter.

For lemma 5.2, the hypothesis $u=a b$ et $v=c$ lead also to $a=\bar{a}=b=\bar{b}=c=\bar{c}$ and we have only the two real solutions. On the contrary, the hypothesis $u=a, v=b c$ leads to $a=\bar{b}=c$ and we have the solution $u=a, v=\bar{a} a$.

- In the inductive case, let us assume that the two lemmas are true when $|u v| \leq n$ and consider two words $u$ et $v$

such that $|u v|=n+1$.

- Lemme 5.1: $u v=\bar{v} \bar{u}$. We compare the lengthes of $u$ et $v$. 
* If $|u|=|v|$, then $u=\bar{v}$.

* If $|u|>|v|$, take $u=\bar{v} u_{1}$. We then also have $\bar{u}=u_{1} v$ hence $u=\overline{u_{1} v}$ and thus $\overline{u_{1} v}=\bar{v} u_{1}$ or $u_{1} v=v \overline{u_{1}}$. By induction hypothesis, the solutions of this equation are $u_{1}=w^{m}, v=w^{n}$ where $w \in R$ which gives $u=w^{m+n}, v=w^{n}$ and $u_{1}=(w \bar{w})^{m}, v=w(\bar{w} w)^{n}$. In that case $u=\bar{v} u_{1}=(\bar{w} w)^{m+n} \bar{w}$ and $v=w(\bar{w} w)^{n}$.

* If $|u|<|v|$, taking $\bar{v}=u v_{1}$, we also have $v=v_{1} \bar{u}$ hence $u v_{1}=\overline{v_{1}} u$. By induction hypothesis, the solutions of this equation are $u=w^{m}, v_{1}=w^{n}$ where $w \in R$ which gives $u=w^{m}, v=w^{m+n}$ and $u=(w \bar{w})^{m} w, v_{1}=(\bar{w} w)^{n}$, which gives $v=(\bar{w} w)^{m+n} \bar{w}$.

- Lemme 5.2: $u v=\bar{v} u$. We compare again the lengthes.

* If $|u|=|v|$, then $u=\bar{v}=v$. (real solutions)

* If $|u|>|v|$, take $u=\bar{v} u_{1}$. We also have $u=u_{1} v$ hence $u_{1} v=\bar{v} u_{1}$. By induction hypothesis, the solutions of this equation are $u_{1}=w^{m}, v=w^{n}$ where $w \in R$ which gives $u=w^{m+n}, v=w^{n}$ and $u_{1}=(w \bar{w})^{m} w, v=(\bar{w} w)^{n}$, which gives $u=(w \bar{w})^{m+n} w$ and $v=(\bar{w} w)^{n}$.

* If $|u|<|v|$, take $\bar{v}=u v_{1}$. Here again we have $v=v_{1} u$, which implies $u v_{1}=\overline{v_{1} u}$. By induction hypothesis, the solutions of this equation are $u=w^{m}, v_{1}=w^{n}$ where $w \in R$ which gives $u=w^{m}, v=w^{m+n}$ and $u=(w \bar{w})^{m} w, v_{1}=\bar{w}(w \bar{w})^{n}$, which gives $v=(\bar{w} w)^{m+n+1}$.

The author thanks Luc Boasson, Maurice Nivat and Laurent Vuillon for their help and support in preparing this paper and also the referee whose remarks and questions were extremely useful.

\section{Author details}

Guy Cousineau

Fédération de Recherche, CNRS-Université de Savoie

Modélisation, Simulation, Interactions Fondamentales, France

\section{References}

[1] D. Beauquier and M Nivat. On translating one polyomino to tile the plane. Discrete and Computational Geometry, 6:575-592, 1991.

[2] H.S.M. Coxeter. Introduction to geometry. John Wiley and sons, 1980.

[3] H.S.M. Coxeter and W.O.J. Mauser. Generators and relations for discrete groups. Springer Verlag, 1972.

[4] M. Gardner. More about tiling the plane: the possibilities of polyominoes, polyamonds and polyhexes. Scientific American, 53:112-115, 1975.

[5] B. Grünbaum and G.C. Shephard. Tilings and patterns. Wiley and sons, 1987. 
[6] Ph. Lechenadec. Canonical forms in finitely presented algebras. Pitman, 1986.

[7] M. Lothaire. Combinatorics on words. Cambridge University Press, 1997.

[8] D. Schattschneider. Will it tile? try the conway criterion! Mathematics Magazine, 53:224-233, 1980.

[9] H.A.G. Vijshoff and J. Van Leeuwen. Arbitrary versus periodic storage schemes and tesselations of the plane using one type of polyomino. Inform. Control, 62:1-25, 1984. 
